\title{
Neurology and Neurotherapy
}

Open Access

CASE REPORT

\section{Missed Cerebral Coil Found in Peripheral Circulation after Endovascular Embolization: A Rare Complication and Review}

\author{
Nissar Shaikh MD, EDIC ${ }^{1}$, Shoaib Nawaz MD ${ }^{2 *}$, Sumayya Aboobacker ${ }^{2}$, Adnan Khan, MBBS ${ }^{3}$, Aisha \\ Alkubaisi, MD ${ }^{4}$, Umais Zaid Momin ${ }^{5}$ and Abdulnasser Thabet, $A^{6}$
}

${ }^{1}$ Senior Consultant SICU, Hamad Medical Corporation, Doha, Qatar

${ }^{2}$ Anesthesiology Resident, Hamad Medical Corporation, Doha, Qatar

${ }^{3}$ Specialist- Department of Neurosurgery, Hamad Medical Corporation, Doha, Qatar

${ }^{4}$ Resident Neurosurgery, Hamad Medical Corporation, Doha, Qatar

${ }^{5}$ DNB, Specialist Radiology, Hamad Medical Corporation, Doha, Qatar

${ }^{6}$ Consultant Neurosurgery, Hamad Medical Corporation, Doha, Qatar

*Corresponding author: Shoaib Nawaz, MD, Anesthesiology Resident, Hamad Medical Corporation, Doha, Qatar, Tel: $+974-50456682$

\begin{abstract}
Endovascular embolization of cerebral aneurysm is common in clinical practice. The rare complication of endovascular embolization is coil misplacement. Most of the reported coil misplacements occur due to premature release of a coil into the cerebral circulation. We report a case of a coil lost into the peripheral circulation due to failed detachment from the micro-catheter at the right place.
\end{abstract}

Case: A young lady without medical history, presented at the emergency department with severe headache. Her consciousness level deteriorated, and her GCS dropped to 5 , requiring immediate intubation, sedation and ventilation. Computer tomography and angiography showed a giant supraclinoid aneurysm of the left internal carotid artery. She was taken for endovascular coiling under general anesthesia and one coil was successfully inserted in the aneurysm. During the insertion of the second coil, it was not possible to detach the coil from the micro-catheter, the coil got stuck at the level of the carotid bifurcation and after several failed attempts to remove it, the coil was swept away into the general circulation and went undetected on fluoroscopy. Next day extremity X-ray showed the coil in the left popliteal artery. Patient was taken for angiography, the misplaced coil was successfully removed and the aneurysm was completely embolized. Her condition improved with supportive care and by day 14 she was successfully extubated and discharged home after another week.

Conclusion: During embolization coil can fail to get detached from the catheter and can go into the peripheral circulation.

\author{
Keywords \\ Aneurysm, Coiling, Carotid artery, Popliteal artery, Missed \\ coil
}

\section{Introduction/Background}

Endovascular embolization therapy of aneurysms started with use of Guglielmi platinum detachable coils (GDC) by electrolysis of the stainless steel delivery catheter [1]. GDC was a game changer and had evolved over last 3 decades to become the standard of care for embolization of aneurysms and fistulas [2]. This procedure is not free from complications, which include perforation, thromboembolism and misplacement. The coils protrusion and misplacement is reported to occur in $0.5 \%$ cases due to premature release into the cerebral circulation with increased risk of ischemic stroke [3]. Failure of detachment of the coil from microcatheter and being lost into the peripheral circulation is not reported in the literature. We report a lost cerebral coil swept away into the peripheral circulation after failed detachement from the micro-catheter inside the aneurysm.

\section{Case}

A 46-year-old healthy woman presented at the

Citation: Shaikh N, Nawaz S, Aboobacker S, Khan A, Alkubaisi A, et al. (2021) Missed Cerebral Coil Found in Peripheral Circulation after Endovascular Embolization: A Rare Complication and Review. Int J Neurol Neurother 8:112. doi.org/10.23937/2378-3001/1410112

Accepted: June 28, 2021: Published: June 30, 2021

Copyright: (c) 2021 Shaikh N, et al. This is an open-access article distributed under the terms of the Creative Commons Attribution License, which permits unrestricted use, distribution, and reproduction in any medium, provided the original author and source are credited. 


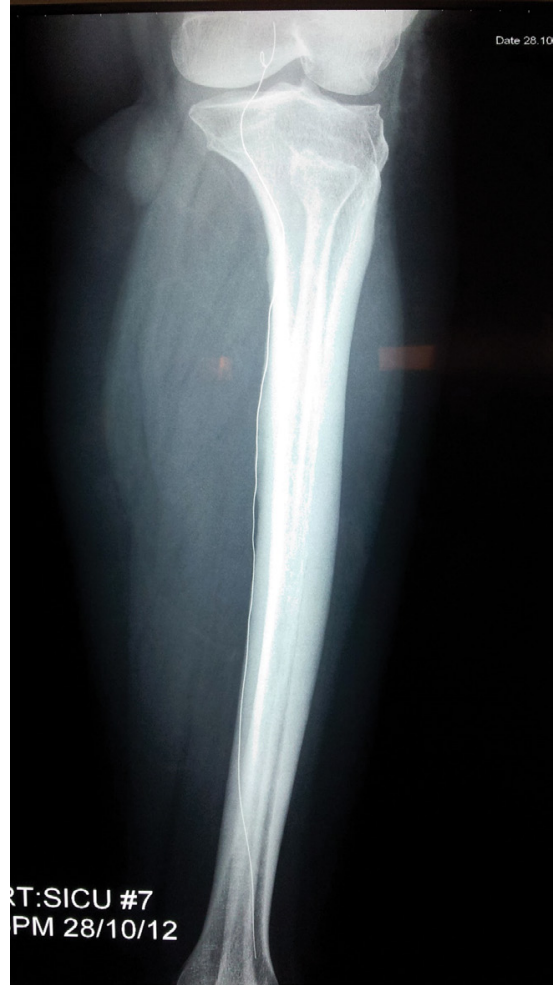

Figure 1: Misplaced coil detected in the left popliteal artery on X-Ray.

emergency department with severe headache. Her medical history was not significant. On examination the patient was in intense pain but obeying commands with tachycardia (102/min) and hypertension (170/92 $\mathrm{mmHg}$ ). She suddenly dropped her consciousness level and Glasgow coma scale (GCS) became 5 requiring intubation, sedation, and ventilation. An urgent computer tomography (CT) brain and CT angiography showed massive subarachnoid haemorrhage with a giant supraclinoid aneurysm of the left internal carotid artery. The patient was transferred to surgical intensive care unit and coiling of the aneurysm was arranged.

After initial resuscitation, the patient went for coiling next day. A micro-balloon catheter was used to block the neck of the aneurysm and a $120 \mathrm{~mm}$ coil was successfully inserted into the aneurysm. Another $120 \mathrm{~mm}$ coil insertion was attempted into the giant aneurysm, but unfortunately the coil could not be detached from the tip of the micro-catheter. Following several failed attempts to detach the coil from the micro-catheter, it was decided to withdraw the microcatheter with the coil. Unfortunately, the coil got stuck at the level of carotid bifurcation. A snare was applied and the coil was successfully brought down to the level of the aortic arch. At this stage, the coil swept out the snare and got lost in the general circulation. Fluoroscopy of head, neck, chest, and abdomen failed to locate the coil. The patient was shifted back to intensive care unit and supportive care and ventilation was continued.

On day 2, extremity X-ray (Figure 1) detected the lost coil in the left popliteal artery. There were no circulation

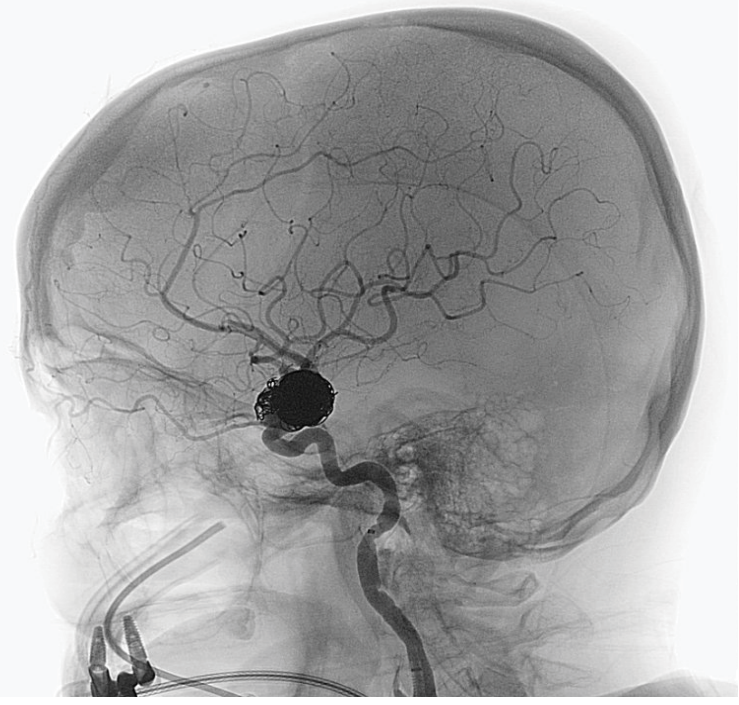

Figure 2: CT angiography showing post coiling of aneurysm.

defects in the left lower limb. On day 3 the patient was taken for cerebral angiography and with the help of micro-balloon catheter the giant cerebral aneurysm was successfully obliterated with multiple coils (Figure 2).

The guiding catheter was withdrawn from the internal carotid artery and advanced into the left superficial femoral artery. Using a $10 \mathrm{~mm}$ snare, the detached coil from the previous procedure was successfully removed from the popliteal artery. The patient was transferred back to the SICU. Enteral feeds, electrolytes correction, sedation and ventilation were continued for another few days as the GCS remained low.

Patient improved neurologically by day 14 . She started to obey simple commands and she was successfully extubated. She was transferred to the ward by day 16 and was discharged home after another week. Followup cerebral angiography showed complete obliteration of the aneurysm and no neurological problems.

\section{Discussion}

Endovascular coiling has become a mainstay in the treatment of ruptured cerebral aneurysms due to obvious benefits which include to be a less invasive procedure and resulting in better clinical outcomes [4]. The main complications of endovascular embolization of cerebral aneurysms are bleeding due to perforation (2.3 to $4.7 \%$ ), thromboembolism ( 2.5 to $14.5 \%$ ), parent artery occlusion (2 to $3 \%$ ), collapse of GDC (8\%) and protruding, migration or misplacement (0.5 to 3\%) [5]. These cerebral coil migrations and misplacements can cause ischemic stroke or other organ ischemia and infarctions [4]. The aneurysm related risk factors for coil protrusion, migration or misplacement are a wide neck and large or very small aneurysms [6]. The reported coil risk factors for migration and misplacement are insertion of small, flexible wires and tightly spiralled ones 
with a diameter of around $1.5 \mathrm{~mm}$ [7]. The majority of cerebral coil misplacements or displacements occur in the cerebral circulation and one case of misplacement in the peroneal artery is reported in the literature [6].

In our patient the coil was difficult to detach from the micro-catheter and during withdrawal the coil got stuck at the carotid artery bifurcation. Several attempts to remove the coil with snare led to losing it in the general circulation. Immediate fluoroscopy search failed to detect the coil in head, neck, thorax and abdomen. Fortunately, we were able to detect the lost coil in the left popliteal artery with X-ray without any embolic complications. In the follow-up angiography, the lost misplaced coil was successfully removed, and complete embolization of the aneurysm was achieved. Patient's follow-up angiography and outpatient clinic visits did not show any abnormality.

\section{Conclusion}

Concluding lines from our case are that coil can fail to detach from the micro-catheter and can get swept away in the general circulation leading to embolization in the peripheral circulation. Early removal of these misplaced coils is the key to avoid thromboembolic complications.

\section{References}

1. Guglielmi G (2018) Guglielmi detachable coils. J Neurointerv Surg 10: e5.

2. Bekelis K, Gottlieb DJ, Su Y, O'Malley AJ, Labropoulos N, et al. (2017) Comparison of clipping and coiling in elderly patients with unruptured cerebral aneurysms. J Neurosurg 126: 811-818.

3. Stidd DA, Johnson AK, Lopes DK (2014) Manual Aspiration Technique to Retrieve a Prematurely Detached Coil during Cerebral Aneurysm Embolization. Neurointervention 9: 2125.

4. Singh DP, Kwon SC, Huang L, Lee WJ (2016) Retrieval of Distally Migrated Coils with Detachable Intracranial Stent during Coil Embolization of Cerebral Aneurysm. J Cerebrovasc Endovasc Neurosurg 18: 48-54.

5. Gatto LAM, Rocha LB, Koppe GL, Demartini Jr Z (2018) Late coil migration after embolization of cerebral aneurysmscase series. Arq Bras Neurocir 37: 71-75.

6. Nikoubashman $\mathrm{O}$, Badenschier R, Müller M, Brockmann C, Schubert G, et al. (2015) Endovascular retrieval of a dislocated coil in the peroneal artery with a stent retriever. BJR 2: 1-2.

7. Abe T, Hirohata M, Tanaka N, Uchiyama $Y$, Morimitsu $H$, et al. (2000) Coil migration, malposition, stretching and retrieval. Interv Neuroradiol 6: 143-147. 